\title{
Il fango e la produzione di biogas: risparmio economico nella gestione degli impianti
}

\author{
Ana Lucía Russo Rinaldi ${ }^{1}$ \\ ${ }^{1}$ Affiliation not available
}

\begin{abstract}
Attualmente, il trattamento e smaltimento dei fanghi di depurazione prodotti dai processi a fanghi attivi comportano costi elevati per gli impianti di trattamento di acque reflue. La necessità di ridurre gli oneri coinvolti in questi processi con alternative sostenibili ha condotto alla ricerca di tecnologie innovative per il pretrattamento dei fanghi. Questo articolo analizza alcune delle opzioni che possono essere implementate. Principalmente si concentra negli effetti dell'utilizzo di trattamenti ad ultrasuoni per migliorare la digestione anaerobica dei fanghi attivi, con conseguente significativa riduzione della produzione di fanghi e un aumento della generazione di biogas come una fonte di energia rinnovabile, il quale può essere utilizzato non solo per compensare $\mathrm{i}$ costi intervenenti nel pretrattamento del fango ma anche per le terze parti. Sebbene studi più approfonditi siano necessari, i benefici economici ed ambientali son evidenti.
\end{abstract}

\section{II carico del trattamento e dello smaltimento dei fanghi di depurazione}

II trattamento e lo smaltimento dei fanghi rappresentano il 50\% dei costi totali dei trattamenti a fanghi attivi negli impianti. La digestione anaerobica si utilizza per la stabilizzazione dei fanghi, la quale risulta in una riduzione della quantità di fanghi e nella produzione di biogas, tuttavia questo processo è un processo lento, il quale comporta tempi di residenza prolungati nelle vasche di digestione, che sono inoltre molto volumetriche. Ciò porta a trovare nuove soluzioni tecnologiche per ridurre la produzione di fanghi attivi. II processo di trattamento di acque reflue a fanghi attivi è uno dei più utilizzati perché l'efficienza del processo è elevata a costi sopportabili. La difficoltà si trova nella generazione di fanghi che devono essere smaltiti, aumentando i costi. Una delle possibili soluzioni proposte dallo studio condotto da Naddeo et al. ${ }^{1}$ propone l'applicazione di un processo innovativo per il pretrattamento con ultrasuoni dei fanghi di depurazione. I 
principali vantaggi del pretrattamento dei fanghi sono l'aumento della solubilizzazione del COD e il miglioramento della biodegradabilità anaerobica, comportando così migliori risultati nella digestione anaerobica dei fanghi $\mathrm{e}$, come conseguenza, la riduzione della quantità di fanghi di depurazione che dovrebbero essere smaltiti. Un altro vantaggio è l'aumento della produzione di biogas risultante da questo processo, il quale può essere utilizzato come fonte di energia per il processo di depurazione. A seguito, l'applicazione a scala reale di questa soluzione alternativa comporterebbe non solo benefici economici ma anche un'ottimizzazione dei processi di depurazione dei fanghi negli impianti di trattamento di acque reflue a fanghi attivi.

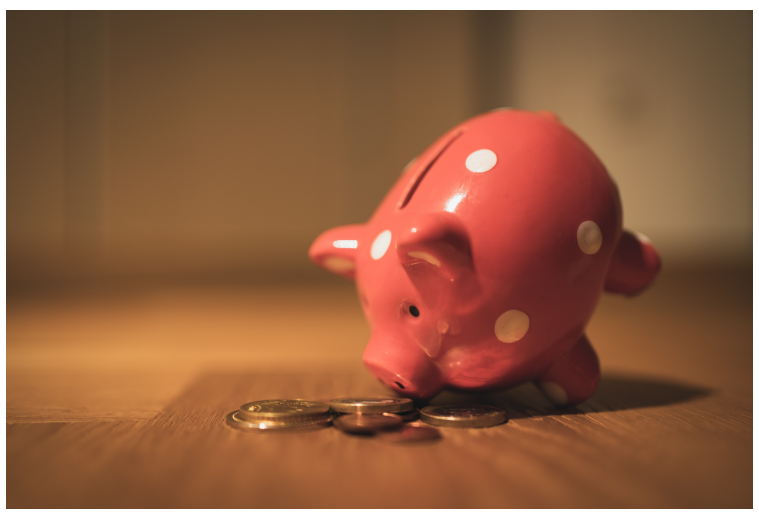

Figure 1: Risparmio economico negli impianti

\section{Soluzioni tecnologiche alternative}

Oltre ai pretrattamenti dei fanghi, si analizzano processi tecnologici alternativi che possono essere applicate per incontrare una soluzione a questa problematica. Secondo l'articolo pubblicato dalla Water Environment Research, "High-rate Anaerobic Side-Stream Reactor (ASSR) Processes to Minimize the Production of Excess Sludge" 2 , si possono sviluppare processi per minimizzare la produzione eccessiva di fanghi durante i trattamenti a fanghi attivi. Questi sistemi sperimentali includono un reattore a flusso laterale anaerobico ad alta velocità e il ricircolo del fango, e hanno dimostrato una qualità accettabile dell'effluente nonostante la produzione minima di fanghi. L'obiettivo di questo studio era dimostrare che l'utilizzo di piccole ASSR diminuisce significativamente la produzione di fanghi. II problema che si presenta, sebbene sia una soluzione adeguata, lo SRT dei fanghi rimane molto lungo. Lo studio si svolge in una vasca di digestione 
anaerobica per indagare il rilascio e degradazione dei fiocchi presenti nel fango per diversi SRT, perché è sospettato che lunghi SRT possano causare una povera flocculazione deteriorando la qualità del flusso. In questo caso, si raggiunge non solo una minimizzazione della produzione di fanghi, ma anche una bioflocculazione effettiva degli stessi. Inoltre, è stato dimostrato che l'implementazione di questi processi ASSR ha avuto successo per alcune applicazioni ma non per tutte. In conclusione, si possono minimizzare le quantità di fanghi prodotti per lo smaltimento attraverso l'incorporazione di piccoli bioreattori anaerobici nei flussi laterali, tuttavia studi più approfonditi devono essere condotti per dimostrare che l'applicazione a scala reale sia possibile.

Un'alternativa diversa per compensare i costi derivati dal trattamento e smaltimento dei fanghi di depurazione sarebbe aumentare la produzione di biogas, il quale può essere utilizzato come fonte sostenibile di energia sia nel proprio impianto di trattamento, sia per essere venduto ad altre aziende ed ottenere così un guadagno economico. La ricerca scientifica ha portato avanti nuove alternative per elevare la produzione di biogas e recenti studi sono stati pubblicati. Uno studio condotto nella Svezia e pubblicato su EMJEI ${ }^{3}$ indaga sulla fattibilità di utilizzare la frazione organica dei rifiuti solidi urbani come co-substrato con fanghi attivi di depurazione per la produzione di biogas. In questo caso, uno degli obiettivi fondamentali è stato aumentare la produzione annuale di biogas nella Svezia. La frazione organica dei rifiuti solidi urbani è un substrato interessante per i processi di digestione anaerobica dovuto alla sua disponibilità, abbondanza e il suo potenziale per produrre biogas. L'ipotesi si basa nella produzione di energie rinnovabile a partire da due fonti principali di rifiuti organici e comporta la co-digestione di fanghi di depurazione con la frazione organica dei rifiuti. Le principali limitazioni includono la necessità di disidratazione dei fanghi per mantenere un tempo di detenzione idraulica sufficiente, ed un consumo maggiore di energia per la miscelazione dovuto alla maggiore viscosità del fango causata dalla co-digestione. Un'altra inquietudine è il rischio di bulking del fango a seguito dell'aumento della viscosità, che possa causare gravi danni agli impianti di biogas. I risultati hanno dimostrato che la co-digestione della frazione organica dei rifiuti solidi urbani con i fanghi di depurazione nei digestori anaerobici è favorabile nonostante le limitazioni segnalate prima, con un potenziale di aumentare la produzione di biogas circa quattro volte senza disturbare la performance del processo.

Lo studio assegnato di Naddeo et al. ha come obiettivo cercare una soluzione effettiva per la riduzione dei costi coinvolti nel trattamento e smaltimento dei fanghi di depurazione. Propone dimostrare che l'applicazione di ultrasuoni nel pretrattamento del fango risulta in benefici in termini economici per quanto riguarda la diminuzione non solo dei fanghi per essere smaltiti ma anche di energia utilizzata per la miscelazione degli stessi, come conseguenza della modificazione della struttura del fango dopo l'applicazione del processo di ultrasuoni che rende il fango significantemente più omogeneo 
e meno sedimentabile. Questi guadagni in termini energetici compensano $\mathrm{i}$ consumi di energia richiesti dai generatori di ultrasuoni. Peraltro, l'aumento nella produzione di biogas può essere utilizzato come fonte di energia per l'impianto e anche per essere venduto alle terze parti. Oltretutto, sostiene che l'applicazione del pretrattamento ad ultrasuoni ai digestori anaerobici riduce i costi in termini di diminuzione del volume dei reattori e della quantità di fango da smaltire, maggiore produzione di energia ottenuta dal biogas generato e minore necessità di agitazione e riscaldamento del fango di depurazione.

\section{L'applicazione di ultrasuoni per la digestione anaerobica}

La digestione anaerobica è la tecnica più applicata per la stabilizzazione dei fanghi di depurazione che risulta nella riduzione di solidi volatili dei fanghi e la produzione di biogas. La ricerca pubblicata sulla Water Science and Technology Journal di A. Tiehm et al. ${ }^{4}$ ha studiato il pretrattamento dei fanghi di depurazione mediante disintegrazione ad ultrasuoni al fine di migliorare la stabilizzazione anaerobica di questi. Le conclusioni di questo studio hanno trovato che il pretrattamento ad ultrasuoni a basse frequenze di ultrasuoni migliora la successiva digestione anaerobica con conseguente migliore degradazione di solidi volatili e una maggiore produzione di biogas. Questo studio supporta che l'applicazione di trattamenti di ossidazione avanzata ad ultrasuoni come pretrattamento per la fase di digestione anaerobica non solo migliora la solubilizzazione di COD e la biodegradabilità anaerobica, ma anche riduce la produzione di fanghi di depurazione e la concentrazione di COD. Peraltro, può essere anche utilizzato nella linea fango con il fine di incrementare la disidratazione dei fanghi, la produzione di biogas e la rimozione di solidi volatili. I fattori che intervengono in questi processi dipendono dalla quantità di energia ad ultrasuoni fornita ai fiocchi di fango.

Altri studi condotti dal SEED ${ }^{56}$, hanno approfondito nell'applicazione di pretrattamenti ad ultrasuoni nella digestione anaerobica di rifiuti solidi urbani per aumentare la produzione di biogas e così ottimizzare il recupero di energia, rafforzando l'ipotesi della applicazione di questo tipo di pretrattamento ai fanghi di depurazione. Per quanto riguarda al trattamento di sonolisi, il suo principale svantaggio è il consumo di energia. I risultati di questi studi possono gettare le basi per lo sviluppo di tecnologie atte alla produzione di grandi quantità di biogas, per la generazione di energia sostenibile e fronteggiare l'elevato consumo di energia del processo. 


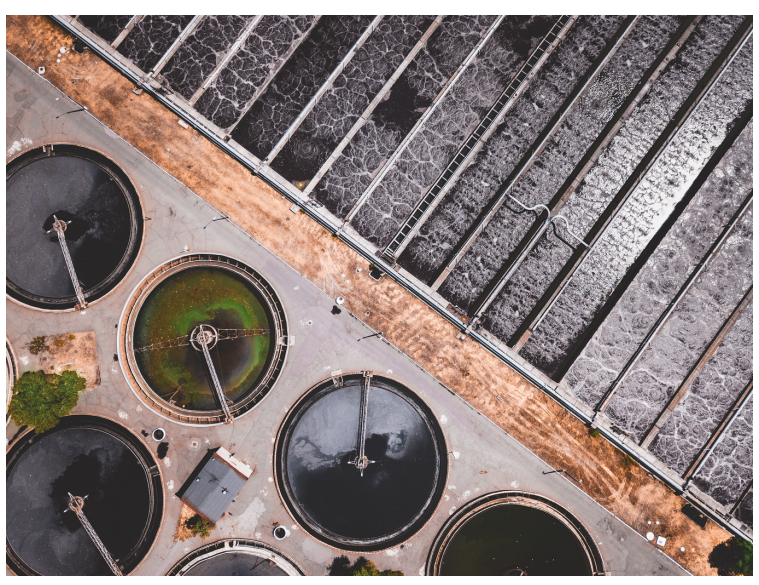

Figure 2: Impianto di Trattamento di Acque Reflue

\section{Conclusione}

In questo lavoro si sono trattati le diversi alternative per affrontare una delle principali problematiche presenti negli impianti di trattamento di acque reflue a fanghi attivi. La necessità di ridurre $i$ costi associati al trattamento e smaltimento del fango di depurazione ha condotto ai ricercatori a trovare soluzioni diverse e l'applicazione di pretrattamenti ad ultrasuoni sarebbe una delle alternative più promettente. I trattamenti ad ultrasuoni sono utili per migliorare la solubilizzazione e biodegradabilità della materia organica, accelerando il processo anaerobico e permettendo il miglioramento della produzione di biogas. Sebbene più ricerche debbano essere condotte per affermare che questa sia la migliore soluzione e sia possibile applicarli su larga scala, i risultati sono ottimistici.

\section{References}

1.Naddeo, V., Belgiorno, V., Landi, M., Zarra, T. \& Napoli, R. M. A. Effect of sonolysis on waste activated sludge solubilisation and anaerobic biodegradability. Desalination 249, 762-767 (2009).

2.Park, C. \& Chon, D.-H. High-Rate Anaerobic Side-Stream Reactor (ASSR) Processes to Minimize the Production of Excess Sludge. Water Environment Research 87, 2090-2097 (2015).

3.Björn, A. et al.. Feasibility of OFMSW co-digestion with sewage sludge for increasing biogas production at wastewater treatment plants. EuroMediterranean Journal for Environmental Integration 2, (2017).

4.Tiehm, A., Nickel, K., Zellhorn, M. \& Neis, U. Ultrasonic waste activated 
sludge disintegration for improving anaerobic stabilization. Water Research 35, 2003-2009 (2001).

5.Cesaro, A. \& Belgiorno, V. Sonolysis and ozonation as pretreatment for anaerobic digestion of solid organic waste. Ultrasonics Sonochemistry 20 , 931-936 (2013).

6.Cesaro, A., Naddeo, V., Amodio, V. \& Belgiorno, V. Enhanced biogas production from anaerobic codigestion of solid waste by sonolysis. Ultrasonics Sonochemistry 19, 596-600 (2012).

7.Errami, M. \& Garner, H. A tale of two citations. Nature 451, 397-399 (2008). 